\title{
UNBOUNDED OPERATORS WITH SPECTRAL CAPACITIES
}

\author{
BY I. ERDELYI
}

Communicated by Robert Bartle, January 15, 1974

The concept of spectral capacity introduced by C. Apostol in [1] and its relationship to decomposable operators [3] established by a theorem of C. Foias [4] are used for an investigation in the unbounded case.

Let $\mathfrak{S}(X)$ denote the family of subspaces (closed linear manifolds) of a Banach space $X$, and let $\mathfrak{F}$ and $\mathfrak{R}$ represent the collection of closed and compact subsets of the complex plane $\pi$, respectively. The superscript c stands for the complement.

1. Definition [1]. A spectral capacity in $X$ is an application $\mathfrak{E}: \mathfrak{F} \rightarrow$ $\mathfrak{S}(X)$ which satisfies the following conditions:

(i) $\mathfrak{E}(\varnothing)=\{0\}, \mathfrak{E}(\pi)=X$;

(ii) $\bigcap_{n=1}^{\infty} \mathfrak{E}\left(F_{n}\right)=\mathbb{E}\left(\bigcap_{n=1}^{\infty} F_{n}\right),\left\{F_{n}\right\} \subset \mathfrak{F}$;

(iii) for every finite open cover $\left\{G_{i}\right\}_{1 \leqq i \leqq m}$ of $F \in \mathfrak{F}, \mathfrak{E}(F)=\sum_{i=1}^{m} \mathbb{E}\left(F \cap \bar{G}_{i}\right)$.

In order to confine the present investigation to densely defined operators on $X$, the following additional constraint on the spectral capacity is needed:

2. Definition. A spectral capacity $\mathbb{E}$ will be referred to as regular if the linear manifold

is dense in $X$.

$$
X_{0}=\{x \in \mathfrak{E}(K): K \in \mathfrak{R}\}
$$

3. Definition. A linear operator $T: D(T)(\subseteq X) \rightarrow X$ is said to possess a regular spectral capacity $\mathfrak{E}$ (abbrev. $T \in \mathfrak{I}(\mathfrak{E})$ ) if it is closed, has a nonvoid resolvent set and satisfies the following conditions:

(iv) $\mathfrak{E}(K) \subseteq \mathfrak{D}(T)$ for all $K \in \mathfrak{R}$;

(v) $T(\mathfrak{E}(F) \cap \mathfrak{D}(T)) \subseteq \mathfrak{E}(F)$ for all $F \in \mathfrak{F}$;

(vi) the restriction $T_{F}=T \mid \mathfrak{E}(F) \cap \mathfrak{D}(T)$ has the spectrum $\sigma\left(T_{F}\right) \subseteq F$, $F \in \mathfrak{F}$.

4. THEOREM. Given $T \in \mathfrak{I}(\mathfrak{E})$. For every $K \in \mathfrak{R}$, the restriction $T_{K}=$ $T \mid \mathfrak{E}(K)$ is a (bounded) decomposable operator on $\mathfrak{E}(K)$ possessing the

AMS (MOS) subject classifications (1970). Primary 47B99; Secondary 47A15, 47B40.

Key words and phrases. Unbounded operators, spectral capacity, decomposable operators, spectral maximal spaces, weak spectral manifolds.

Copyright (c) American Mathematical Society 1974 
spectral capacity $\mathfrak{E}_{K}$ defined by

$$
\mathfrak{E}_{K}(F)=\mathfrak{E}(K \cap F) \text { for all } F \in \mathfrak{F} .
$$

In the proof it is shown that $T_{K}$ is bounded by the closed graph theorem and $\mathfrak{E}_{K}$, as defined by (1), is a spectral capacity for $T_{K}$.

A property which is instrumental for the subsequent study of operators in $\mathfrak{F}(\mathfrak{E})$ is expressed by the following

5. TheOrem. Let $T \in \mathfrak{I}(\mathfrak{E})$ and $K \in \mathfrak{R}$. The following statements are equivalent:

(i) $x \in \mathfrak{E}(K)$;

(ii) there exists an $X$-valued function $\tilde{x}$ analytic on $K^{\mathrm{c}}$ satisfying the equation

$$
(\lambda-T) \tilde{x}(\lambda)=x \quad \text { for all } \lambda \in K^{\mathrm{c}} .
$$

The implication (i) $\Rightarrow$ (ii) of the proof is based on the single-valued extension property of a decomposable operator. (ii) $\Rightarrow$ (i) is proved first for an $x \in X_{0}$ with the help of a result by C. Foiaş [4]:

$$
\left\{y \in \mathbb{E}(L): \sigma_{T_{L}}(y) \subseteq K\right\}=\mathbb{E}(K) \quad \text { where } L(\supset K) \in \Re .
$$

Next, for $x \notin X_{0}$, the density of $X_{0}$ in $X$ and the closeness of $\mathbb{E}(K)$ complete the proof.

6. THEOREM. Every $T \in \mathfrak{I}(\mathfrak{E})$ has a unique regular spectral capacity.

In the first stage of the proof, the application of Theorem 5 shows that any two regular spectral capacities $\mathbb{E}$ and $\mathfrak{E}_{1}$ of $T$ agree on $\mathcal{H}$. Next the property expressed by Definition 2 implies that $\mathfrak{E}(F)=\mathfrak{E}_{1}(F)$ for all $F \in \mathfrak{F}$.

7. THEOREM. For every $K \in \mathfrak{R}, \mathfrak{E}(K)$ is a spectral maximal space of $T \in \mathfrak{I}(\mathfrak{E})$.

The proof is performed with the help of Theorems 4 and 5 .

8. TheOREM. Given $T \in \mathfrak{I}(\mathfrak{E})$. For every $x \in X$ there exists a nonvoid open set $U \subset \pi$ and a sequence $\left\{\tilde{x}_{n}\right\}$ of $X$-valued functions analytic on $U$, with

$$
\lim _{n \rightarrow \infty}(\lambda-T) \tilde{x}_{n}(\lambda)=x \quad \text { for all } \lambda \in U
$$

Again, the proof is obtained by an application of Theorem 5 .

We redefine E. Bishop's concept of weak spectral manifold $\mathfrak{N}(F, T)$ [2, Definition 2] without the restriction of $T$ being bounded as follows: Given $T: \mathfrak{D}(T)(\subseteq X) \rightarrow X$ and $F \in \mathfrak{F}, \mathfrak{N}(F, T)$ is the set of all $x \in X$ which 
have the property that for each $\varepsilon>0$ there exists an $X$-valued function $\tilde{x}$ analytic on $F^{\mathrm{c}}$ such that $\|x-(\lambda-T) \tilde{x}(\lambda)\|<\varepsilon$, for all $\lambda \in F^{\mathrm{c}}$.

A straightforward consequence of Theorem 8 is the following

9. Corollary. Given $T \in \mathfrak{I}(\mathfrak{E})$. For every $F \in \mathfrak{F}$,

$$
\mathfrak{E}(F)=\mathfrak{N}(F, T) .
$$

\section{REFERENCES}

1. C. Apostol, Spectral decompositions and functional calculus, Rev. Roumaine Math. Pures Appl. 13 (1968), 1481-1528. MR 40 \#3333.

2. E. Bishop, $A$ duality theorem for an arbitrary operator, Pacific J. Math. 9 (1959), 379-397. MR 22 \#8339.

3. C. Foiaş, Spectral maximal spaces and decomposable operators in Banach space, Arch. Math. 14 (1963), 341-349. MR 27 \#2865.

4. - Spectral capacities and decomposable operators, Rev. Roumaine Math. Pures Appl. 13 (1968), 1539-1545. MR 40 \#3334.

Department of Mathematics, Temple University, Philadelphia, Pennsylvania 19122. 